\title{
Variación diatópica del español americano a través de recursos hipermedia
}

\section{Dr. Mário Domingues Ferreira da Cruz¹, Inst. Politécnico do Porto y CIDTFF, Portugal}

Recibido:

12 de abril, 2017

Aceptado:

2 de octubre, 2017

\section{Resumen}

Hoy es casi imperativo poner atención al español de Hispanoamérica en la clase de E/LE, a causa del dinamismo económico y cultural de la región. Por ello, es posible concordar con Anadón Pérez (2003) cuando refiere que se debe valorar un modelo de enseñanza y aprendizaje holístico con usos panhispánicos, desarrollando alumnos aspirantes a ser plurilingües dentro de una lengua histórica.

Los entornos de trabajo actuales requieren no solo conocimientos sólidos sobre temas específicos, sino también diferentes capacidades, que incluyen la reflexión crítica, la autodirección, las habilidades interculturales, etc. Por consiguiente, existe la necesidad de replantear la forma en que se ha estado enseñando idiomas. De hecho, parece pertinente trabajar las variedades lingüísticas y culturales del español latinoamericano (Moreno Fernández, 2000) en la clase de español como lengua extranjera (ELE), teniendo en cuenta una pedagogía de los discursos que involucra interactivamente a los sujetos en el análisis y producción de textos (Fonseca, 1992).

En este artículo se pretende presentar un estudio de caso relacionado con las variedades lingüísticas y culturales de Hispanoamérica, y dos propuestas didácticas que plasman dichas variedades mediante recursos hipermedia (cuentos, cómics, cortometrajes, películas y juegos digitales, entre otros), planteadas con alumnos portugueses universitarios en Oporto. Se tuvo en cuenta un análisis previo de los conocimientos del alumnado y los manuales de enseñanza usados en el contexto, respecto a las variedades del español. Los cuestionarios de autoevaluación que los alumnos resolvieron al final demuestran que ellos desarrollaron las competencias plurilingüe y pluricultural.

\section{Abstract}

\section{Diatopic variation of American Spanish through hypermedia resources}

It is imperative to pay attention to Hispanoamerican Spanish in the E/LE class due to the economic and social dynamism of the region. For this reason, it is possible to agree with Anadón Pérez (2003) when referring to the necessity of valuing a holistic learning and teaching model with Panhispanic uses, developing students with plurilingual aspirations within a historic language. Current working environments require not only solid knowledge regarding specific topics, but also different abilities that include critical thinking, self-learning, intercultural skills, etc. As a consequence, there is a need of rethinking the way we have been teaching languages.

In fact, it seems appropriate to work the linguistic and cultural variations of Latin-American Spanish (Moreno Fernández, 2000) in the Spanish as a Foreign language (SFL) class, considering a pedagogy of the discourse that involves interactively the subjects in the analysis and production of texts (Fonseca, 1992). This article aims to present a case study related to the linguistic and cultural variations of Hispanoamerica, and two didactic proposals that implement these variations through hypermedia resources (short stories, comics, short films, movies and digital games, among others) in Portuguese university students in Oporto. Students' previous knowledge was considered along with the teaching manuals used in the context regarding Spanish variations. The self-evaluation questionnaires answered by the students at the end show a development of plurilingual and pluricultural competencies.

1 Este trabajo cuenta con el apoyo financiero de los Fondos Nacionales a través de la Fundación para la Ciencia y la Tecnología (FCT), I.P., en el marco del proyecto UID/CED/00194/2013 .

\section{(6) FCT cidtff}

2 Mario Domingues es Doctor en Didáctica y Formación, graduado de la Universidad de Aveiro, Portugal. Está haciendo otro doctorado en Estudios Lingüísticos en la Universidade de Vigo, España. Es licenciado en Enseñanza de Inglés y Alemán y tiene tres maestrías, tanto en Didáctica de Lenguas como en Enseñanza de Inglés y Español para primaria y Enseñanza de Inglés y Español para secundaria, obtenidas en distintas universidades. Labora como profesor e investigador de la Escuela Superior de Educación del Instituto Politécnico do Porto, Portugal y también es investigador en el CIDTFF, Universidad de Aveiro. Contacto: mariocruz@ese.ipp.pt.
Mario Domingues Ferreira da Cruz. Variación diatópica del español americano a través de recursos hipermedia. Revista Comunicación. Año 38 , volumen 26 , número 2 , juliodiciembre, 2017. Instituto Tecnológico de Costa Rica. ISSN: 0379-3974 / e-ISSN1659-3820.
PALABRAS CLAVE:

Lengua española, enseñanza de lenguas, América Latina, uso didáctico del ordenador, elaboración de medios de enseñanza, lingüística aplicada.

\section{KEY WORDS:}

Spanish tongue, Languages learning, Latin America, didactive use of the pc, creation of teaching methods, applied linguistics. 


\section{INTRODUCCIÓN}

La globalización puede generar efectos paradójicos, pues influye en el plano cultural al crear a la vez homogeneización y heterogeneidad en la sociedad. De hecho, los medios de comunicación con sus mensajes ricos contribuyen a la creación de una cultura de mercado. Pero también se da la adaptación de los contenidos de dichos mensajes al contexto de una región o sea, se experimenta la llamada glocalización (Regueiro Rodríguez, 2016 y Jurado, 2016).

Se puede decir que hay una tendencia a la desaparición de las fronteras, una creciente necesidad de una movilidad laboral y un desarrollo de necesidades de comunicación plurilingües y pluriculturales en un mercado globalizado. El inglés ya no es suficiente como lengua franca, por lo que se siente cada vez más la presencia de una diversificación lingüística, tanto en términos de la existencia de numerosos idiomas en la comunicación internacional -sea física o digital- como en cuanto a las variedades dentro de un mismo idioma (Cruz, 2011 y 2015).

Asimismo, debe repensarse el aprendizaje de idiomas; es decir, hace falta modificar el modo de abordar la enseñanza de las lenguas, orientando esta última hacia la dimensión intercultural y plurilingüe (Paricio, 2004). El posicionamiento del español como lengua internacional y el papel destacado que los pueblos hispanoamericanos tienen en la vitalidad y proyección del idioma en el mundo globalizado (Mora-Figueroa, 1998) son cada vez mayores, por lo que se cree es necesario valorar los estudios relacionados con la pertinencia de la inclusión de las variedades lingüísticas y culturales del español hispanoamericano en el proceso de enseñanza-aprendizaje.

En este artículo, por un lado, se explicará el componente teórico del estudio de caso, teniendo en cuenta los avances que se han dado respecto a las investigaciones relacionadas con el tema de las variedades lingüísticas y culturales del español latinoamericano, la sociolingüística variacionista, la formación panhispánica del profesor y los nuevos abordajes y recursos didácticos auténticos. Por otro lado, se presentarán propuestas didácticas panhispánicas basadas en el uso de recursos literarios e hipermedia, implementados en un estudio de caso con alumnos lusohablantes de los niveles A2/B1 (85 alumnos) en una universidad portuguesa. Para validar las propuestas didácticas planteadas los alumnos contestaron cuestionarios de autoevaluación, dando cuenta de lo que aprendieron.

\section{De la cohesión a la heterogeneidad pragmática del español}

Los lingüistas de hoy están de acuerdo en que las lenguas son vivas, naturales, abiertas y sufren cambios y por lo tanto, sus hablantes se expresan de manera diferente utilizando el mismo idioma, por lo que lengua y variación son dos conceptos indisociables (Alkmim, 2001 y Camacho, 2001). Se sabe que la sociolingüística variacionista contribuye a entender que el español -al igual que otros idiomas- es un sistema abierto, a través del cual sus hablantes pueden expresarse de manera distinta (Alkmim, 2001, Camacho, 2001 y Regueiro Rodríguez, 2009).

Respecto al español, muchos de los estudios analizados coinciden en que no hay un español preferible, un español "más correcto", pues esta visión ignora la riqueza del idioma y sus variantes. Por otra parte, esta perspectiva basada en el mito de la homogeneización lingüística puede ser perjudicial para la enseñanza, ya que permite excluir características importantes de un idioma histórico como el español. En otras palabras, es necesario desmitificar esta aparente superioridad de determinada variedad lingüística sobre las demás (Antunes, 2003 y Rosenblat, 1984).

Aunque se hable de una enorme diversidad en relación con el español, diferentes lingüistas prefieren recalcar su homogeneidad y acentuar su carácter de koiné, que permite una cierta unidad, homogeneidad, la cual facilita la comunicación entre todos los hispanohablantes (Moreno Fernández, 2000). La verdad es que no se puede olvidar que "la intercomprensión entre un mexicano y un santiagueño, un zaragozano y un limeño es mucho más sencilla" (Marcos Marín, 2001, pp. 70-73) que la que se da entre hablantes de distintas variedades del inglés, por ejemplo.

Asimismo, es sabido que el español es una de las lenguas "más conexionadas entre las grandes, sin variantes dialectales ininteligibles que apunten hacia la fragmentación, y fonéticamente muy clara (...)" (Salvador, 2001). Autores como Lapesa (1980) o Roña (1965) están de acuerdo en destacar la existencia de un continuo de comprensión existente en el idioma 
Variación diatópica del español americano a través de recursos hipermedia

Tabla 1. Rasgos generales del español de Hispanoamérica

\section{Rasgos léxicos Rasgos fonéticos Rasgos morfosintácticos}

- Arcaísmos, palabras que en Es- • Seseo, pronunciación de z como • Voseo o sea, el uso del pronompaña suenan arcaicas. $\quad S$

- Indigenismos provenientes de los • Yeísmo, pronunciación de II liar en lugar de tú. idiomas precolombinos. como y.

- Extranjerismos, sobre todo angli- - Rotacismo, confusión de r-y l. $\quad$ gunda persona del plural. cismos.

- Aspiración de h- inicial proce- • Posposición de posesivos, como dente de f- latina y de la j caste- en la hija mía.

llana.

- Gran utilización del sufijo apre-

- Relajación o pérdida de las consonantes implosivas (seguidas de otra consonante, en medio de una palabra) y finales. ciativo diminutivo, incluso en adverbios (ahorita).

- Pérdida de -d-intervocálica.

- Uso de sufijos: (-ada, -ida) como en muchachada, platicada.

- Ausencia de leísmo, laísmo o loísmo.

español, pues consideran que las diferencias dentro del territorio americano extenso son mínimas dentro de la estructura del habla total. No obstante, esta variación se debe a las diferencias lingüísticas que los colonos llevaron desde la península al mestizaje (Sueiro Justel, 2011) y más tarde a la influencia del sustrato (lenguas amerindias) y también al superestrato posterior (los esclavos africanos o la migración italiana en Argentina, por ejemplo) (Lapesa, 1980), lo que hace que el español sea una lengua mestiza, un código lingüístico que representa una síntesis de todas estas culturas y los contactos entre todos los idiomas (Torres Solórzano, 2010 y Konieczna-Twardzikowa, 2002).

Según los estudios analizados (Moreno Fernández, 2010; Alexa Izquierdo y Utrilla, 2010; Vaquero, 1995, 1996; Alvar, 1978; Pfander, 2009; Calvo, 2005; Merma Molina, 2004 y Tesoro, 2013), se han identificado los siguientes rasgos diatópicos léxicos, fonéticos y morfosintácticos de la región hispanoamericana.

El estudio de la región de Hispanoamérica es relevante debido no solo a la herencia cultural de la región que "es Europa transferida y América trasvasada" (Alvar, 2002, p. 251), sino también a la creación e implementación de uniones -como el Mercado Común del Sur (MERCOSUR) o la Asociación Latinoamericana de Integración (ALADI) - y la atracción turística que ofrece al mundo. Por esta razón, resulta imposible descuidar el gran dinamismo de la región en la clase de ELE y por lo tanto, parece importante trabajar "las variedades lingüísticas que pueden encontrar los extranjeros que visitan Hispanoamérica, los referentes culturales que van a marcar las relaciones interpersonales" (Alvar, 2002, p. 251).

El desconocimiento de las diferencias diatópicas entre el español de las regiones hispanoamericanas y el de la región ibérica puede ocasionar algunos infortunios comunicativos, sobre todo respecto al léxico y la pragmática (Regueiro Rodríguez, 2016). De hecho, no es posible confundir cohesión de la lengua con homogeneidad de usos lingüísticos y pautas pragmáticas de comunicación, pues hay diferencias fundamentales que pueden ocasionar infortunios comunicativos (Regueiro Rodríguez, 2016). Además, el profesor de español debería desarrollar conocimientos sobre estos rasgos, por lo que a continuación se analiza cómo se pueden Ilevar a la clase de ELE, de acuerdo con estudios realizados.

\section{El abordaje panhispánico en el aula de ELE}

El autor concuerda con Anadón Pérez (2003) cuando dice que se debe apostar por un modelo de enseñanza holístico de usos panhispánicos, que sea "capaz de dar respuesta a usos geográficos y sociales distintos desde un punto de partido común (...)", de manera que los alumnos se sientan "plurilingües dentro de una lengua histórica, es decir, a hablar de forma diferenciada según las circunstancias y los niveles de habla". 
El encuentro de los alumnos con el lenguaje y culturas de las variedades del español hispanoamericano fácilmente se consigue a través de entornos físicos o virtuales de naturaleza intercultural (e inevitablemente plurilingüe) y de la manipulación de recursos reales multisensoriales. La idea es aumentar la eficacia de la comunicación pragmática del alumno en un contexto específico (Bortoni-Ricardo, 2005) y al mismo tiempo, marcar y hacer sentir la identidad cultural y social del hablante nativo de la variante lingüística de la lengua meta.

Asimismo, los alumnos lusohablantes que estudian español, igual que otros alumnos europeos, revelan interés por las variedades hispanoamericanas del español por: a) su riqueza lingüística, incluso su exotismo por el mestizaje propio de la región; b) necesidades profesionales en el futuro, pues muchos quieren trabajar o buscar trabajo en el área; c) atracción por el conocimiento de la geografía, historia, música, cine, pero sobre todo literatura hispanoamericana del boom y post-boom (Altmann y Vences, 2004; Cruz y Saracho, 2016 y Cruz, 2015).

En el proceso de enseñanza y aprendizaje, el profesor de ELE tiene que estar bien preparado, ya que no basta tener conocimientos de planificación, metodologías de enseñanza, léxico, gramática y fonética (Pastor Cesteros, 2016). De hecho, un profesor de español debe demostrar respeto ante la cultura de los países hispanohablantes y la del país de la sociedad en la que vive y trabaja, siendo consciente de su papel como intermediario intercultural y sacando el máximo provecho de los recursos tecnológicos y audiovisuales en el trabajo con la cultura en las clases de ELE.

También, resulta casi imperativo que los futuros profesores estén preparados científica y pedagógicamente respecto a los conocimientos de las variedades lingüísticas y culturales del español de la región de Hispanoamérica. No obstante, como refiere Moreno Fernández (2010), para el profesor de ELE en un contexto heteroglósico "es obligado conocer cuáles son esas variedades y reflexionar sobre cuáles merecen considerarse variedades dialectales o geolectales aplicables en la enseñanza" (p. 162). En otras palabras, debe tratar de enseñar las variedades hispanoamericanas que considera más importantes para sus alumnos o sea, hay que preguntarles directamente lo que saben, lo que quieren aprender y para qué, a través de cuestionarios creados con este fin (Altmann y Vences, 2004; Cruz y Saracho, 2016 y Cruz, 2011, 2015).

En este sentido, el docente debe tener una preparación científica y lingüística que le permita orientar a los alumnos según sus necesidades, para que puedan actuar adecuadamente y con plena competencia gramatical en los diferentes contextos sociales de los países hispanohablantes (Andión Herrero y Burgman, 2013 y Andión Herrero, 2008). Por otra parte, es necesario que el profesor combata los peligros que conllevan las visiones estereotipadas sobre los países hispanohablantes, dando oportunidad a los alumnos de construir sus propios juicios sobre las culturas del diasistema español, a través del estudio de las variantes lingüísticas y sobre todo del léxico y aspectos fraseológicos (Moreno García, 2000 y Llamas y Martínez, 2001).

La verdad es que en la sociedad globalizada actual es posible tener en un aula diferentes identidades dialectales, por lo que el profesor tendrá que respetarlas para que se evite lo que Flórez Márquez (2000) Ilama "situaciones extremas en las que a veces se escuchaba al profesor (...) decirles a sus alumnos que no se dice papas sino patatas, o corregirlos en su pronunciación si seseaban (...)" (p. 311). Asimismo, no se puede controlar que los alumnos accedan al léxico o estructuras gramaticales, morfológicas y sintácticas propias de otras variedades, ya que están en contacto con otras culturas y pueblos a través de Internet, por ejemplo.

Teniendo en cuenta el entorno de enseñanza y aprendizaje en Portugal, el manual de enseñanza aún se destaca como uno de los tipos de materiales más importantes e influye en las prácticas de enseñanza y aprendizaje en la clase de ELE. Muchos autores como Rodrigues (2005) y Pontes (2009) señalan que los manuales deberían proporcionar más oportunidades para la interacción con situaciones comunicativas experienciales de los contextos lingüísticos y culturales de las variantes del español (Pontes, 2009; Cruz, 2015 y Cruz y Saracho, 2016), pues se verifica un "etnocentrismo, a veces de forma inconsciente" (Cerdeira yVicente, 2009, p. 369) o una exotización de dialectos o culturas determinadas, lo que condiciona el "acercamiento a la riqueza cultural del español" 
(Cerdeira y Vicente, 2009, p. 369). De hecho, los distintos países son representados en los manuales de ELE de una manera poco equilibrada, siendo "evidente que la información sobre España ocupa el primer lugar (...) y que los países latinoamericanos, en cambio, son abordados en menor medida (...)" (Andrade, 2011, p. 35).

El trabajo con las variedades lingüísticas del español no es una tarea fácil, debido a la escasez de materiales didácticos creados para este fin. Pero el profesor no puede solamente basar sus prácticas en el libro, por lo que es conveniente que utilice otros materiales. De esta manera, hay que evaluar en qué medida el material auténtico representa las realidades nacionales, regionales y locales de las variantes con relación a su calidad y relevancia en el contexto de aprendizaje y necesidades futuras del alumnado. Por lo tanto, es importante que el docente fomente un contacto significativo que vaya más allá de la exploración de curiosidades relativas al vocabulario o expresiones (Cruz y Saracho, 2016) o sea, se deben desarrollar actividades en clase que permitan al alumnado una explotación y reflexión lingüística y cultural (Coan, y Pontes, 2013).

Asimismo, para quien escribe, es imprescindible integrar los contenidos culturales en las tareas de aprendizaje, "intentando enlazar lo cultural y lo lingüístico" y "relacionar las manifestaciones culturales de distintas áreas (literatura, pintura, música, cine, teatro, danza, etc.) para que el alumno construya una suerte de mapa cognitivo al que podrá ir integrando elementos nuevos" (Lerner, 2000).

Es opinión del autor que el enlace de lo lingüístico con lo cultural se consigue a través del estudio de diferentes tipos de discursos, es decir, por una parte textuales, más relacionados con la literatura y por otra, con ambientes digitales y multimedia, a través de "la siempre valorada narrativa, la en ocasiones olvidada poesía, la no siempre bien aprovechada música, las poco explotadas artes plásticas, y como no, las TIC" (Mercau, 2014, p. 7). De hecho, diversos autores como Moreno Fernández (2010) creen que se deben seleccionar materiales de enseñanza que sirvan como "muestras vivas de una variedad determinada" (p. 161), haciendo uso de los medios electrónicos que proporcionan muestras del español (hablado, escrito, cantado, etc.) de muchos lugares del mundo hispano.

Hay que resaltar aquí el uso de las obras literarias, pues estos recursos pueden ser aliados del profesor en el trabajo con las variedades, "(...) cubriendo necesidades lingüísticas (conocimientos de gramática, sintaxis, léxico, etc.) y culturales (conocimientos geográficos, históricos, gastronómicos, etc.)" (Mercau, 2014, pp. 20-21). No obstante, no se puede también olvidar el rol de los espacios virtuales, donde los estudiantes encuentran un espacio para comunicarse en la lengua meta (Coello, 2011), pues los entornos virtuales ofrecen al alumnado otras experiencias y posibilidades de desarrollo de tareas de trabajo comparativo de los modos de hacer y comprender el mundo.

Asimismo, teniendo en cuenta la heterogeneidad lingüística de Hispanoamérica, resulta imperativo analizar la actualización del idioma en los discursos, para ver los usos que sus hablantes realizan, en los cuales asumen un papel y estatus en la praxis social y en las relaciones de unos con los otros. Por consiguiente, el análisis e interpretación de los discursos permitirá observar las experiencias de vida y las interpretaciones que cada sujeto hace del discurso real (Sueiro Justel, 2011). Según Fonseca (1992, p. 236), esta pedagogía de los discursos supone que cada sujeto puede acceder a ellos como instrumentos de descubrimiento de uno mismo, de los otros y del mundo, a través de una experiencia interactiva.

Se dan a conocer a continuación las características de este estudio y su contexto, para luego prestar atención a algunas propuestas didácticas construidas para dar respuesta a lo hablado en estos capítulos.

\section{El proyecto: propuestas didácticas panhispánicas}

El autor coincide con investigadores como Fernández-Corbacho (2014), Mora (2013) y Knutson (2003), quienes consideran que la experiencia, por la dimensión afectiva que contiene, deberá funcionar como una brújula que permitirá al alumno orientar su aprendizaje con una fuerte relación con el mundo real y virtual. De hecho, quien escribe considera que el enfoque comunicativo experiencial es lo más efectivo en la implementación del estudio de las variedades lingüísticas y culturales en la clase de ELE, ya que "supone la conexión de los contenidos 
de lengua con el estudiante, sus aficiones e intereses y con su experiencia del mundo (...)" (FernándezCorbacho, 2014, p. 1), para que el alumno participe en un proceso interactivo de co-construcción de "conocimientos culturales sobre las sociedades de los países hispanohablantes y la suya propia, analizando sus semejanzas y diferencias" (Valls Campà, 2011, p. 16), adquiriendo habilidades que le permitan adquirir los conocimientos por sí mismo e interaccionar satisfactoriamente con hablantes nativos.

Este proyecto se centró en las variedades hispanoamericanas del español (Liceras, Carballo y Droege, 1995; Beaven y Garrido, 2000) que plasman el binomio cohesión-diversidad, recurriendo a discursos literarios e hipermedia con aportes de la sociolingüística histórica. Asimismo, el estudio se desarrolló alrededor de los objetivos específicos y cuestiones de investigación que se encuentran en la tabla 2.

Con el fin de ser capaz de alcanzar los objetivos propuestos y obtener respuestas a las preguntas anteriores, la perspectiva metodológica investigativa adoptada estuvo encuadrada en el paradigma etnográfico y de naturaleza cualitativa. La combinación de diferentes técnicas y herramientas de análisis culminó en una triangulación metodológica y de fuentes con el objetivo de contrastar e interpretar los datos obtenidos y darles credibilidad a los resultados (cf. Anadón Pérez, 2003; Altmann y Vences, 2004).

Tabla 2. Objetivos y cuestiones de investigación

\section{Objetivos específicos \\ Cuestiones de investigación}

A. Describir las representaciones y saberes sociolingüísticos y socioculturales que los profesores $y$ alumnos tienen sobre las variedades lingüísticas y socioculturales de Hispanoamérica, respecto al proceso de enseñanza de ELE en instituciones de educación superior.

B. Inventariar las representaciones que los profesores de instituciones de educación superior tienen de las prácticas de enseñanza que favorezcan la (re)construcción de saberes relacionados con el español y sus variedades.

C. Inventariar los cursos que ofrezcan contenidos so- 2. ¿Cómo son trabajadas las variedades lingüísticas bre Hispanoamérica en instituciones de educación superior portuguesas, respecto a la formación del profesorado.

1. ¿Qué representaciones tienen los alumnos y los profesores de las variedades lingüísticas y culturales del español latinoamericano en la enseñanza superior en Portugal?

D. Analizar manuales de enseñanza de ELE respecto al 3 . abordaje de contenidos relacionados con las variedades lingüísticas y socioculturales del español de Latinoamérica.

E. Crear propuestas didácticas basadas en los discursos literarios e hipermedia que plasman las variedades lingüísticas y culturales de Hispanoamérica, teniendo en cuenta un enfoque comunicativo experiencial.

F. Evidenciar la creación de nuevas representaciones y saberes sociolingüísticos y socioculturales por los estudiantes, cuando son estimulados a través de y culturales del español hispanoamericano en los cursos de formación de profesores en Portugal?

prácticas basadas en un enfoque comunicativo experiencial. 
El corpus principal de este trabajo se basó, por un lado, en el análisis de discursos literarios e hipermedia, respecto a las variedades lingüísticas y culturales del español latinoamericano que dichos recursos ofrecen y por otro, en el análisis de los cuestionarios, manuales, notas de campo de reflexión crítica sobre las prácticas y también de las fichas de trabajo y los cuestionarios de autoevaluación producidos por una muestra de 85 estudiantes lusohablantes de enseñanza superior de ELE de los niveles A2/B1 de la región de Oporto, en Portugal.

Asimismo, se aplicaron distintos tipos de instrumentos de recolección de datos con diferentes funciones. Primero, se procedió a la preparación y aplicación de un cuestionario con un enfoque en los aspectos sociobiográficos para inventariar las representaciones sociolingüísticas y socioculturales de los estudiantes sobre los idiomas y su aprendizaje, y su relación con el mundo hispánico (https://goo.gl/forms/ FlhtPtsGrCG2w5p22). De igual manera, se verificó la importancia de inventariar las percepciones que tienen los profesores sobre la valoración de dichas variedades en la enseñanza superior y qué tipo de actividades o estrategias preparan para trabajarlas en clase. Asimismo, se estudió qué tipo de preparación brindan los cursos de graduación y de posgrado en Portugal a los futuros profesores de ELE respecto al contenido "Hispanoamérica" (https://goo.gl/ forms/0Ndj89N2wjTqEUwM2). A su vez, se prestó atención a las representaciones que se encuentran en los manuales de enseñanza de ELE. No cabe en este artículo presentar y analizar estos datos, pero sería pertinente resaltar que: a) se comprobó un interés por parte de los estudiantes portugueses en las variedades del español, que es reconocido y compartido por sus profesores, los cuales todavía no las dominan de manera más compleja; b) los docentes no están preparados para trabajar en la clase de ELE, teniendo en cuenta un abordaje panhispánico; c) los manuales de ELE presentan una visión lingüística y cultural eurocéntrica, y tampoco contienen muestras vivas de las culturas hispanoamericanas, por consiguiente, no dan cuenta de la riqueza de las variedades, presentándolas solamente como mera información.

Después de haber analizado los cuestionarios iniciales, los cursos de formación docente en Portugal y los manuales, se eligieron algunos recursos literarios e hipermedia que parecieron adecuados para trabajar, teniendo en cuenta las reales necesidades del alumnado y profesorado, y estudios previos, como por ejemplo los de Altmann y Vences (2004), Anadón Pérez (2003) y Cruz (2015).

Una vez presentado el estudio, es importante destacar que es en estos recursos donde se pondrá la atención a partir de ahora y a presentarlos, ya que ese el otro objetivo de este artículo: presentar dos propuestas didácticas de materiales relacionados con muestras vivas de las culturas de los países hispanoamericanos, a través de los cuales los alumnos se pueden involucrar en su estudio de las variedades del español, que fueron implementados en las clases. Después de la presentación de cada una de las propuestas, se hará un análisis de algunos de los resultados de los cuestionarios de autoevaluación reIlenados por los alumnos al final de cada secuencia didáctica.

\subsection{Las medias de los flamencos}

En esta secuencia didáctica se propuso trabajar las variedades del Río de la Plata y andina a través de distintos tipos de recursos: un cuestionario inicial, un cuento y su guión de lectura, el diccionario de la Real Academia Española (DRAE), vídeos del YouTube, la aplicación Heads Up! y catálogos de ropa en línea.

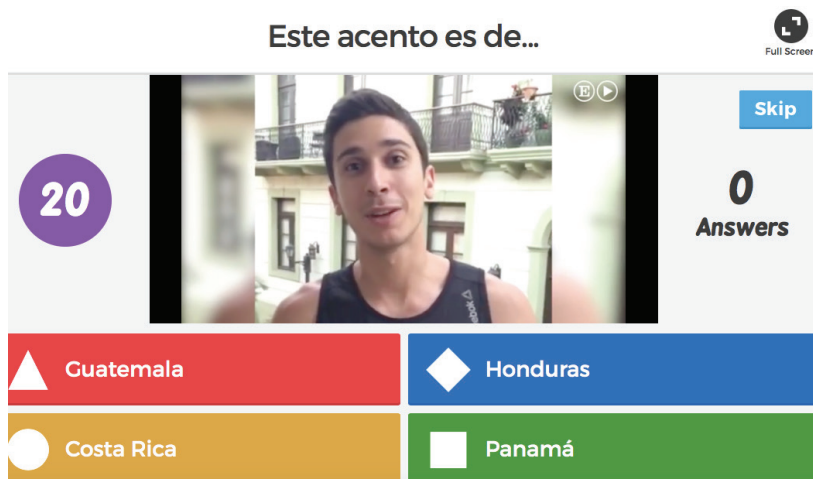

Imagen 1. Ejemplo de pregunta sobre contenidos lingüísticos en el Kahoot! “¿Cuánto sabes de Hispanoamérica?

Fuente: https://play.kahoot.it/\#/k/468cbd0c-9da9-4fa9-a5469cc3a51624d2

Esta tarea gamificada (Fernández-Corbacho, 2014) ayudó a inventariar sus dificultades, pues la plataforma grababa sus respuestas, por lo que es posible analizarlas en plenaria o simplemente guardarlas como elementos de evaluación. 
El tango es la música más característica de Argentina y...

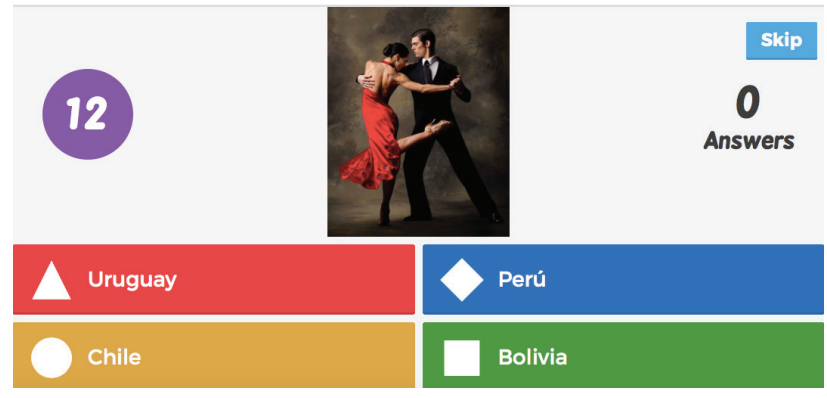

Imagen 2. Ejemplo de pregunta sobre contenidos culturales en el Kahoot! "¿Cuánto sabes de Hispanoamérica?

Fuente: https://play.kahoot.it/\#/k/468cbd0c-9da9-4fa9-a5469cc3a51624d2

De igual manera, se pretendió desmitificar los prejuicios que el alumnado ha tenido sobre la región latinoamericana y pensado sobre los rasgos lingüísticos de la región (Antunes, 2003; Cruz, 2015; Cruz y Saracho, 2016), incluyendo el desconocimiento de la coexistencia del español y las lenguas amerindias.

En seguida, después del calentamiento y preparación del alumnado para la incursión en Hispanoamérica, los alumnos empezaron por ver un vídeo del centro comercial sponsor (https://www.youtube.com/ watch? $v=$ IXUVOrNUhjA) y se activó el vocabulario necesario para el tema de la clase y la lectura del cuento. Los alumnos tenían oportunidad de revisar las prendas de vestir de una manera divertida y tomar contacto con la variedad andina, con relación al acento y expresiones coloquiales (;Qué bacán!, hacer lo bien sin mirar a quien, etc.) Se revisó el léxico relacionado con las prendas de vestir con la aplicación Heads Up! para sistemas Android (https://play.google.com/store/apps/details?id=com. wb.headsup\&hl=pt) o iOS (https://itunes.apple.com/ us/app/heads-up/id623592465?mt=8). En seguida, los alumnos crearon un mapa mental con el léxico que ya conocían en el sitio web www.goconqr.com (ver ejemplos en la imagen 3 ).

En seguida, en plenaria se leyó el cuento Las medias de los flamencos de Horacio Quiroga, escritor uruguayo y durante la lectura se hicieron algunas actividades: a) ejercicios de verdadero o falso, b) preguntas de comprensión un poco más complejas, c) ejercicios de búsqueda de significado de las palabras y su origen en el DRAE, d) ejercicios de correspondencia entre verbos y sus significados, e) ejercicios de búsqueda de palabras para ampliación del vocabulario

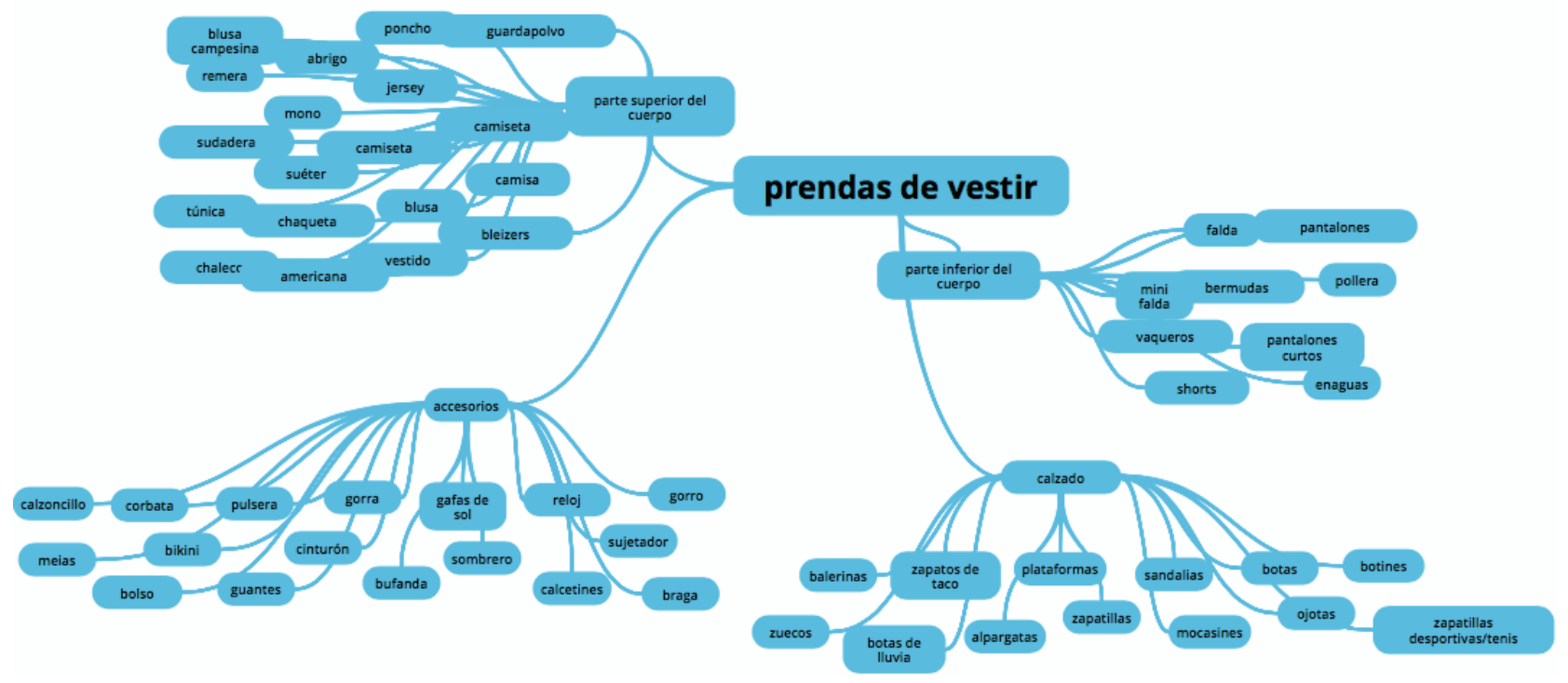

Imagen 3. Ejemplo de un mapa mental creado por los alumnos

Fuente: https://www.goconqr.com/pt/p/8122433-prendas-de-vestir-mind_maps 
relacionado con las prendas de vestir y f) el estudio

de los diferentes significados de la palabra "pollera" (véase la imagen 4).

\section{E. Hay vocabulario relacionado con prendas de vestir. Subraya ese léxico. \\ F. Una de las palabras es pollera. Consulta su definición aquí: http://definicion.de/pollera/ y completa la tabla siguiente:}

\begin{tabular}{|c|c|c|}
\hline Expresión & Significado & Países \\
\hline \multicolumn{3}{|l|}{$\begin{array}{l}\text { "La pollera pasó toda la } \\
\text { mañana con los pollos } \\
\text { trabajando" }\end{array}$} \\
\hline \multicolumn{3}{|l|}{$\begin{array}{l}\text { "Esta noche me voy a estrenar } \\
\text { la pollera rosa" }\end{array}$} \\
\hline \multicolumn{3}{|l|}{$\begin{array}{l}\text { "Tenemos que ir a la pollera a } \\
\text { comprar unos pollos para la } \\
\text { cena del sábado" }\end{array}$} \\
\hline $\begin{array}{l}\text { "Actualmente los polleros } \\
\text { cobran por lo general de } 3000 \text { a } \\
7000 \text { dólares por cruzar la } \\
\text { frontera de los EE.UU" }\end{array}$ & & \\
\hline
\end{tabular}

Imagen 4. Ejemplo de ejercicios de comprensión en la ficha de trabajo

Después de la lectura, los alumnos tuvieron la oportunidad de (re)crear el cuento usando los sitios StoryBoard.that (https://www.storyboardthat.com) o PowToon (https://www.powtoon.com), en los cuales elaboraron un cómic o un vídeo (dibujo animado) imaginando un encuentro entre los flamencos y la lechuza después de la fiesta, una vez que esta los engañó. Con este ejercicio se estimula la creatividad de los alumnos, ya que tenían la oportunidad de relacionarse con el texto de otra manera (Mercau, 2014 y Fonseca, 1992). En el ejemplo siguiente los alumnos crearon un nuevo final de la historia en un vídeo.

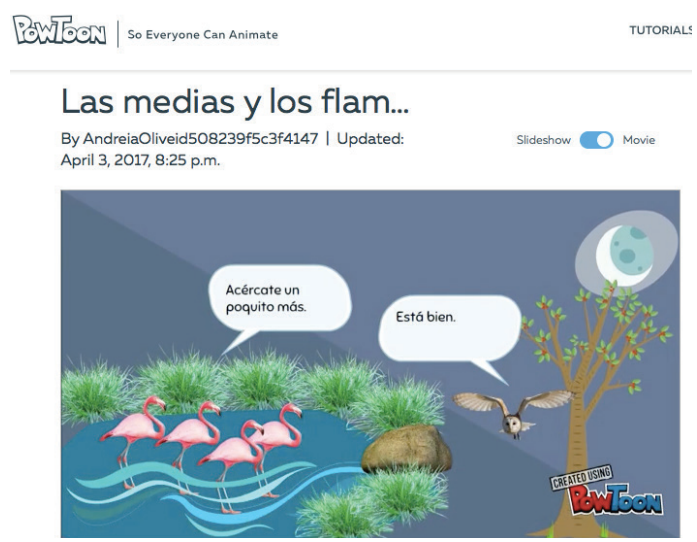

Imagen 5. Vídeo creado por los alumnos con un nuevo final para el cuento.

Fuente: https://www.powtoon.com/m/bvgG7kL4pt9/1/m
Como tarea final, los alumnos también podían ampliar sus mapas mentales, los cuales crearon en el sitio web www.goconqr.com, añadiendo nuevas palabras que encontraron en los catálogos de ropa de las regiones andina, caribeña, centroamericana o rioplatense: http: //www.sabrina.com.ar, http://www. patprimo.com y https://www.porporacr.com. Tuvieron contacto con distintas variedades al mismo tiempo, de manera que pudieron comprobar la homogeneidad y diversidad propias del español (Regueiro Rodríguez, 2016 y Jurado, 2016).

Prestando atención al análisis de los cuestionarios de autoevaluación planteados (https://goo.gl/forms/ A2SVcPmvU0xk5vLy1), solo $38 \%$ de los alumnos consideraron que no eran capaces de decir palabras/ expresiones relacionadas con prendas de vestir de una de las regiones hispanoamericanas. Los restantes alumnos dieron ejemplos de palabras/expresiones, como por ejemplo: "remeras (ar), sácon (ar), guayaberas (CO), medias (Co)" (AL93). Sin embargo, pocos (20\%) consiguieron dar ejemplos con expresiones.

De igual manera, gran parte de los alumnos lograron describir de qué trataba el cuento Las medias de

$3 \quad$ Los ejemplos de respuestas de los alumnos que se presentan a partir de ahora están codificados con la sigla AL y el número del alumno. Se eligieron algunas respuestas que mostraron una buena comprensión de la tarea. 
los flamencos (69\%). Se eligió un ejemplo de todas las respuestas, que daba cuenta de la comprensión efectiva del cuento: "Las víboras organizaron una fiesta. Los flamencos querían adornarse con medias pero nadie tenía medias como las que ellos deseaban. Los flamencos fueron a visitar la lechuza por las medias. Ella les dio unas hechas con piel de víbora. Luego los flamencos fueron muy populares en la fiesta hasta que las víboras notaran lo que eran los adornos. Las víboras mordieron los flamencos y desde entonces todos los flamencos tienen las patas coloradas (AL12)".

Respecto a la cuestión "soy capaz de comprender el significado de la palabra 'pollera' en distintos contextos", 84\% de los alumnos contestaron afirmativamente y dieron algunos ejemplos como los siguientes: "Pollera puede ser una falda tradicional e algunos países hispanoamericanos. También puede ser el lugar donde se crían los pollos" (AL14) o "Persona que se encarga del traslado ilegal de personas. Persona que cuida de los pollos. Una falda" (AL19).

\subsection{El avión de la bella durmiente}

En esta secuencia didáctica los alumnos fueron expuestos a diferentes variedades al mismo tiempo. Los grandes objetivos de la clase era la comprensión del cuento El avión de la bella durmiente de Gabriel García Márquez, para trabajar el tema del aeropuerto y los viajes.

Se introdujo el tema de los viajes a través del análisis de algunos cómics de Mafalda. De esta manera los alumnos empezaron a tener contacto con el voseo. Siguiendo con las actividades de prelectura, los alumnos tenían la oportunidad de buscar información sobre el autor y hacer algunos ejercicios que activaran el vocabulario necesario para la lectura efectiva del cuento de García Márquez. Es importante resaltar que los alumnos pudieron ver algunos vídeos de compañías latinoamericanas sobre los pasos que se debe seguir cuando se llega al aeropuerto, incluyendo los trámites de seguridad (ver imagen 6). Se trabajó el léxico siguiente: boleto/ticket, mostrador, asiento de ventanilla, panel informativo, facturar el equipaje, etc.

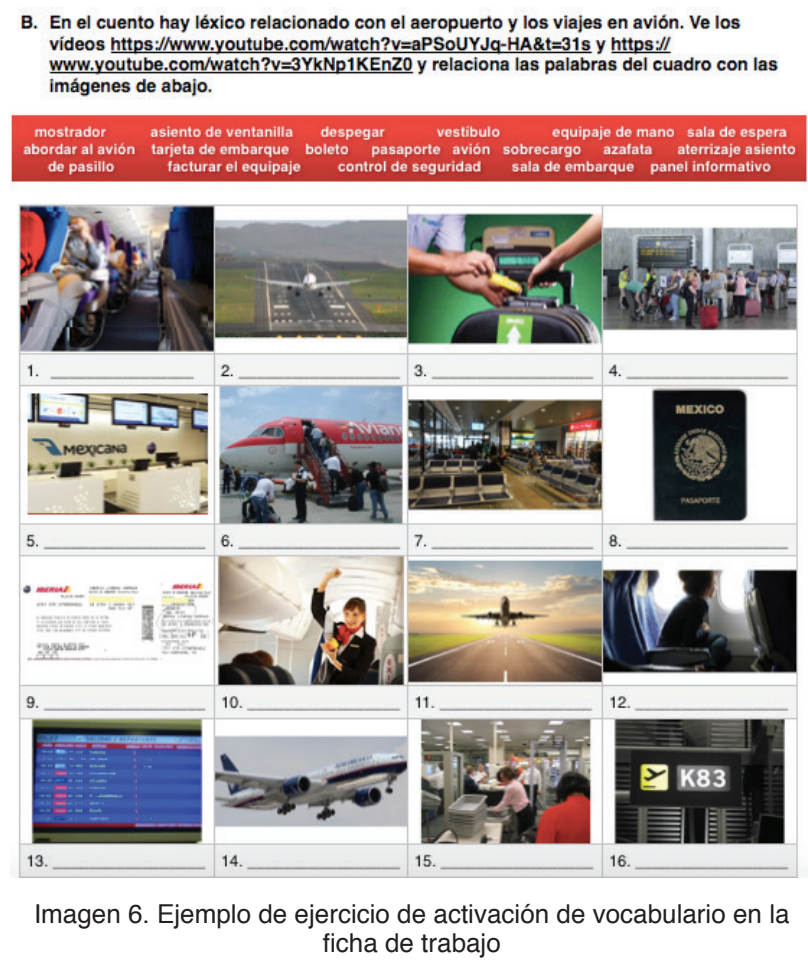

En seguida se leyó el cuento y los alumnos hicieron ejercicios de comprensión, pero con una reflexión lingüística y cultural (Coan y Pontes, 2013): a) ejercicios de verdadero o falso, b) ejercicios de recolección de palabras o expresiones de descripción de los personajes, c) ejercicios de búsqueda del significado de palabras en el DRAE (aura, bugambilias, trancos, destello, diferidos, enseres, rebatiña, penumbra, fogonazos, crines, etc.), d) ejercicios de búsqueda de palabras en el texto más utilizadas en Latinoamérica (almuerzo, computadora, bella, muchachas, etc.) y e) ejercicios sobre el significado de expresiones fraseológicas (me da lo mismo, hasta que Dios quiera, a tu salud, carajo, etc).

Respecto a la gramática, se pudo revisar los tiempos verbales del pasado (pretérito perfecto simple, pretérito perfecto compuesto y pretérito imperfecto), ya que el texto contiene muchos ejemplos de los tres casos. Fue posible pedirle a los alumnos que buscaran en el texto ejemplos sobre dichos tiempos verbales y sus marcadores, por ejemplo (cf. Mercau, 2014).

Para consolidar el tema de los viajes se puede trabajar el fragmento uno de la película argentina Relatos Salvajes, para lo cual se puede (re)activar el vocabulario necesario para la comprensión del vídeo con una consulta al blog https://www.diariodelviajero. 
com/tag/etimologias-viajeras, donde los alumnos tienen la posibilidad de descubrir la historia detrás de las palabras boleto, billete, chárteres, chequear, maleta, petaca, visa, visado, así como rellenar una tabla con información acerca del origen de las palabras, los países donde son utilizadas y sus distintos usos.

Después de ver el vídeo, mediante ejercicios de verdadero o falso, se verificó la comprensión. Luego se pidió a los alumnos que se concentraran en la conversación entre los viajeros protagonistas:

- "Usted, ¿qué hace?

- Me mataste.

- ¿Por qué?

- $\quad$ Ese usted me hundió hasta el quinto círculo del infierno. Tengo que hacerme un entretejido urgente. (...)

- ¿Ahora la querés arreglar? (...)

- Vos elegí el término que quiera y yo lo instalo en la comunidad artística. (...)

- ¿Lo seguís viendo?"

Se solicitó a los alumnos que se enfocaran en los pronombres personales y los verbos, subrayándolos. Además, se les preguntó qué rol tenían los pronombres personales en la conversación. Fácilmente los alumnos llegaron al fenómeno lingüístico voseo. Después, se les pidió que analizaran los siguientes artículos: ¿Por qué algunos países de América Latina usan el 〈vos〉 en vez del ‘tú'? (http://www.bbc.com/ mundo/noticias-america-latina-36928497), Acerca de vós e de vocês (https://ciberduvidas.iscte-iul. pt/consultorio/perguntas/acerca-de-vos-e-de-voces/29509) y Até que o vós me doa (http://visao.sapo. pt/opiniao/ricardo-araujo-pereira/2016-12-15-Ateque-o-vos-me-doa). Estos artículos explican el uso y el origen del vos y del você en portugués. De manera contrastiva, los alumnos comprendieron el origen, rasgos y usos geográficos de los dos fenómenos en español y portugués (ver imagen 7). Gracias a esto, se va un poco más allá de la exploración de curiosidades o exotización de las variedades, pues los alumnos entenderán la importancia que tiene el voseo en el mundo hispanohablante (cf. Cruz y Saracho, 2016) y aumentarán su eficacia comunicativa en posibles contactos con latinoamericanos de esta región (cf. Bortoni-Ricardo, 2014).

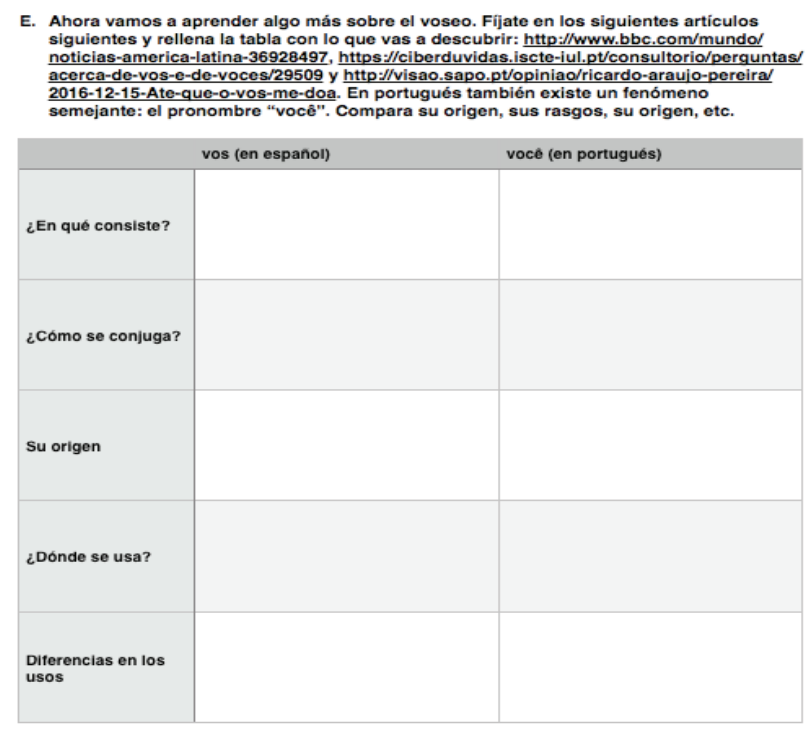

Imagen 7. Ejemplo de la tabla que los alumnos rellenan sobre el vos y el você en la ficha de trabajo

Para trabajar las características fonéticas de la variedad del español argentino, los alumnos vieron el fragmento de nuevo y apuntaron ejemplos que escucharon para cada uno de los rasgos fonéticos, como se ve en la imagen siguiente:

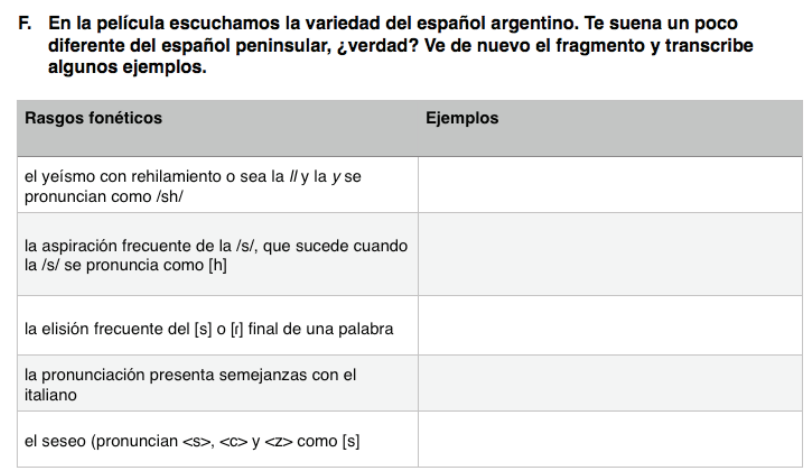

Imagen 8. Ejercicio sobre los rasgos fonéticos en la ficha de trabajo

Con respecto al análisis de los cuestionarios de autoevaluación (https://goo.gl/forms/pr1f2SuiG2ZfKoOl2), la gran mayoría de los alumnos (61\%) confirmaron que aprendieron vocabulario relacionado con los viajes en español de la región hispanoamericana, dando ejemplos de dos palabras en español que suelen ser más utilizadas en América Latina: "visa, boleto, chequear, petaca" (AL8), "camión, boleto" (AL23) y "Guaguas" (AL25).

De igual manera, demostraron que comprendieron el cuento El avión de la bella durmiente $(71 \%$ del 
alumnado). En esta respuesta el alumno entendió el cuento: "La historia de un hombre que ha quedado varado en el Aeropuerto Charles de Gaulle de París mientras espera su vuelo a la ciudad de New York. En la fila para los controles de pasajeros ve a una mujer, a quien describe como una bella mujer con ojos de color almendra. No puede dejar de contemplarla. Unas ocho horas después, cuando finalmente aborda el avión, tras ubicarse en su asiento, sorprendido descubre a la mujer hermosa sentada en el asiento de al lado. Ella se toma dos pastillas y duerme todo el viaje. El hombre espera que despierte en algún momento para entablar conversación, cosa que finalmente no ocurre" (AL9).

Lo mismo sucede con el fragmento de la película Relatos Salvajes, ya que $72 \%$ de los alumnos consiguieron resumirlo en cinco frases, como en este ejemplo de uno de los alumnos: "Una modelo va viajar de avión. En el avión conoce un hombre que es un cazador de talentos. Después ellos descubren que conocen la misma persona, Gabriel Pasternak. En el avión están más personas que conocen a ese hombre y que es él que está pilotando el avión. El avión empieza a cair por culpa del piloto que quiere venganza" (AL7).

Respecto al voseo, fenómeno lingüístico algo complejo, $65 \%$ de los alumnos lograron explicar qué es el voseo, pero solamente $20 \%$ dijeron dónde se usa y dieron cuenta de algunos ejemplos, como los siguientes: "Se denomina voseo al uso del pronombre vos en sustitución del pronombre tú. Se usa en Latinoamérica. Ejemplos: Vos sos idéntico. Vos tenés su misma cara" (AL3) o "Es conocido por voseo el uso del pronombre vos como forma de tratamiento informal en Argentina, por ejemplo. Ejemplos: ¿Vos trabajás muchas horas? ¿Vos podés abrir la ventana?" (AL7).

Estas secuencias didácticas contienen muestras vivas lingüísticas y culturales (Lerner, 2000) de las variedades del español de una manera integrada y con ellas se busca involucrar el alumnado en su análisis lingüístico y cultural, teniendo en cuenta propuestas panhispánicas que no valorizan unas variedades más que otras (Antunes, 2003 y Rosenblat, 1984).

\section{CONSIDERACIONES FINALES}

El autor cree que el aprendizaje de ELE debe ser un encuentro para la acción comunicativa, el diálogo intercultural, la maximización de las múltiples habilidades de los estudiantes (García, 2010) y de una relación de afectividad con el lenguaje (Marques, 2004), permitiendo la creación de situaciones de interacción, recepción e interpretación de diversos tipos de recursos, con respecto a una variedad de tipos de textos y el uso de un lenguaje hipermedia (Consejo de Europa, 2001), como los que se encuentran representados en este artículo.

De hecho, como se vio, parece que una variedad textual da respuesta a lo recomendado por Mercau (2014), pues son textos auténticos que permiten acceder a los modos de hablar, sentir y relacionarse de los hablantes nativos de las variedades hispanoamericanas del español. A través de la pedagogía de los discursos, los alumnos pueden interactuar con y recrear los textos, descubren los rasgos lingüísticos y culturales de las distintas variedades del español, y al mismo tiempo construyen una visión amplia y plurilingüe de la lengua y cultura meta que estudian.

Es opinión de quien escribe que es necesario implicar a los alumnos en tareas con documentos reales y en contacto con las variedades lingüísticas y culturales del español para contribuir a su competencia plurilingüe, dentro del mismo idioma. A través de los datos recogidos se pudo verificar que los alumnos son capaces de interactuar con los textos, centrando su atención en cuestiones que les despiertan alguna motivación e interés.

Asimismo, el estudio de las variedades lingüísticas y culturales del español se puede hacer en el aula, teniendo en cuenta: a) ejercicios de búsqueda de información en diccionarios en línea, diarios o artículos, b) actividades de tratamiento de información a través del uso de aplicaciones y herramientas Web 2.0, como Kahoot!, c) tareas de análisis crítico de obras literarias, ricas en contenidos culturales vivos y con voces de actores sociales propios de las culturas espejadas, d) muestras de vídeo que dan a conocer contextos de interacción propios de los pueblos, sus sociedades y culturas, y e) trabajos de proyecto, de naturaleza interdisciplinaria. 
Variación diatópica del español americano a través de recursos hipermedia

\section{REFERENCIAS BIBLIOGRÁFICAS}

Alexa Izquierdo, M., y Utrilla, J. (2010). La lengua española en América: normas y usos actuales. Valencia: Universidad de Valencia.

Alkmim, T. (2001). Sociolingüística. En F. Mussalim y A. Bentes (Orgs.), Introdução à lingüística: Domínios e fronteiras (pp. 21-47). São Paulo: Cortez.

Altmann, W., y Vences, U. (2004). América latina en la enseñanza del español. ¿Encuentro o encontronazo? Theorie und Praxis des moderen Spanischunterrichts. Berlin: Edition Tranvia.

Alvar, M. (1978). Dialectología hispánica. Madrid: UNED.

Alvar, M. (2002). Español en dos mundos (Temas de Hoy). Madrid: Alianza Editorial.

Anadón Pérez, M. (2003). Hispanoamérica y el español de América en la enseñanza del español como segunda lengua entre alumnos ingleses (Disertación de Máster). Universidad de Barcelona: Barcelona. Recuperado de http://www. mecd.gob.es/redele/Biblioteca-Virtual/2005/ memoriaMaster/1-Semestre/ANADON-P.html

Andión Herrero, M. (2008). La diversidad lingüística del español: la compleja relación entre estándar, norma y variedad. Actas de/ VIII Congreso de Lingüística General. El valor de la diversidad [meta] lingüística. Recuperado de http://www.lllf.uam. es/clg8/actas/pdf/paperCLG10.pdf

Andión Herrero, M. y Gil Burgman, M. (2013). Las variedades del español como parte de la competencia docente: Qué debemos saber y enseñar en ELE/L2. Actas del I Congreso Internacional de Didáctica de Español como Lengua Extranjera. Recuperado de http://cvc.cervantes.es/ensenanza/biblioteca ele/publicaciones centros/PDF/ budapest 2013/06 andion-gil.pdf

Andrade, V. (2011). La presentación de los países hispanohablantes en los libros de texto de español como lengua extranjera. Un estudio sobre libros de texto usados en el bachillerato sueco y sobre la perspectiva del profesorado. Estocolmo: Universidad de Estocolmo. Recuperado de http:// www.diva-portal.org/smash/get/diva2:473270/ FULLTEXT01.pdf
Antunes, I. (2003). Muito além da gramática. Por um ensino de línguas sem pedras no caminho. São Paulo: Parábola.

Beaven, T. y Garrido, C. (2000). El español tuyo, el mío, el de aquél... ¿Cuál para nuestros estudiantes? Actas del XI Congreso Internacional ASELE. Recuperado de http://cvc.cervantes.es/ensenanza/biblioteca_ele/asele/pdf/ 11/11_0181.pdf

Bortorni-Ricardo, S. (2005). Educação em Língua Materna: a Sociolinguística na sala de aula. São Paulo: Parábola.

Calvo, J. (2005). Sobre préstamos léxicos del quechua al español (desde el entorno peruano). En C. Hernández Alonso y L. Castañeda (Eds.), El español de América: actas del VI Congreso Internacional de "El español de América". Valladolid: Diputación de Valladolid.

Camacho, R. (2001). Sociolingüística parte II. En F. Mussalim y A. Bentes (Orgs.), Introdução à lingüística: Domínios e fronteiras (pp. 49-75). São Paulo: Cortez.

Cerdeira, P. y Vicente, J. (2009). Etnocentrismo y variedades dialectales en el aula ELE. En A. Barriento, J. Martín, V. Delgado y M. Fernández. (Eds.), El profesor de español LE-L2. Actas del XIX Congreso Internacional de la Asociación para la Enseñanza del Español como Lengua Extranjera (pp. 363-370). Cáceres: Universidad de Extremadura.

Coan, M. y Pontes, V. (2013). Variedades lingüísticas e ensino de espanhol no Brasil. Revista Trama, 9(18), 179-191. Recuperado de e- revista.unioeste.br/index.php/trama/article/download/8252/6079

Coello, P. (2011). Las nuevas tecnologías en el aula de ELE: Los blogs de grupo como herramienta de aprendizaje colaborativo. Actas del IV Congreso Internacional: La enseñanza del español en un mundo intercultural. Jornadas pedagógicas. Santiago de Compostela: Universidad de Santiago de Compostela. Recuperado de http://www. mecd.gob.es/dctm/redele/Material- RedEle/ Numeros\%20Especiales/2012_ESP_13_IVCongreso\%20FIAPE/ 2012_ESP_13 _07Concheiro. pdf?documentld=0901e72b812ee7b3 
Consejo de Europa. (2001). Marco común europeo de referencia para las lenguas: aprendizaje, enseñanza, evaluación. Madrid: Instituto Cervantes, Anaya, Ministerio de Educación Cultura y Deporte.

Cruz, M. (2011). Consciência cultural crítica numa comunidade virtual educativa de línguas (Tesis de Doctorado, Universidade de Aveiro, Aveiro, Portugal). Recuperado de http://repositorio.esepf. pt/handle/123456789/1487

Cruz, M. (2015). O lugar da Hispanoamérica no processo de ensino-aprendizagem de Espanhol como Língua Estrangeira no Ensino Secundário Português (Disertación de Máster, Universidad de Aveiro, Aveiro, Portugal).

Cruz, M. y Saracho, M. (2016). Materiales para la enseñanza-aprendizaje de las variedades lingüísticas y culturales de Hispanoamérica en la clase de ELE. Ponencia presentada en el XXVII Congreso Internacional de ASELE, La Rioja.

Fernández-Corbacho, A. (2014). Aprender una segunda lengua desde un enfoque comunicativo experiencial. Programa de Desarrollo Profesional. Madrid: Editorial Edinumen.

Flórez Márquez, Ó. (2000). ¿Qué español enseñar? o ¿Cómo y cuándo enseñar los diversos registros o hablas del castellano? En ¿Qué español enseñar?: norma y variación lingüísticas en la enseñanza del español a extranjeros. Actas del XI Congreso Internacional ASELE (pp. 311-316).

Fonseca, J. (1992). Ensino da língua materna como pedagogia dos discursos. En Linguística e Texto/ Discurso. Teoria, Descrição, Aplicação (pp. 235248). Lisboa/Nice: Ministério da Educação/ Universidad de Nice.

García, M. (2010). Multisensory learning applied to TEFL in secondary education. Madrid: Universidad Complutense de Madrid.

Jurado, M. (2016). La variedad mexicana del español ante los desafíos de la globalidad. Conferencia presentada en el XXVII Congreso Internacional de ASELE, La Rioja.

Knutson, S. (2003). Experiential learning in SecondLanguage Classrooms. TESL Canada, 20, 52-64.
Konieczna-Twardzikowa, J. (2002). El español, ¿lengua del mestizaje didáctico y la intraculturalidad? Comunicación presentada en el XIII Congreso de ASELE. Recuperado de https://cvc.cervantes.es/ ensenanza/biblioteca_ele/asele/pdf/13/13_0491. pdf.

Llamas, C., y Martínez, C. (2001). La diversidad estilística: una propuesta para su tratamiento en el aula de ELE. Recuperado de http://cvc.cervantes.es/ ensenanza/biblioteca_ele/asele/pdf/11/11_0481. pdf.

Lapesa, R. (1980). Historia de la lengua española. Madrid: Gredos.

Lerner, I. (2000). La clase es un tango. Recuperado de https:// www.edinumen.es/index. php?option=com_content\&view=article\&id=83 \& catid $=11$ \&ltemid $=51$.

Liceras, J., Carballo, A. y Droege, S. (1995). El tema de las variedades del español en los programas de español como lengua extranjera. Revista de Filología Románica, 11/12, 291-308.

Marcos Marín, F. (2001). De lenguas y fronteras: el espanglish y el portuñol. Nueva Revista, marzoabril, 70-79.

Marques, L. (2004). O aproveitamento didáctico da canção na aula de língua estrangeira. A Página da Educação, 130. Recuperado de http:// www.apagina.pt $/$ ? aba $=7 \& \mathrm{cat}=130 \& \mathrm{doc}=9878 \& \mathrm{mid}=2$

Mercau, C. (2014). La cultura hispanoamericana en las clases de ELE: nuevas perspectivas y aplicaciones. Oviedo: Universidade de Oviedo. Recuperado de http://digibuo.uniovi.es/dspace/handle/10651/28151

Merma Molina, G. (2004). El contacto lingüístico en el español andino peruano. Estudios pragmáticocognitivos. Alicante: Universidad de Alicante.

Mora, F. (2013). Neuroeducación. Solo se aprende aquello que se ama. Madrid: Alianza Editorial.

Mora-Figueroa, S. (1998). El peso de la lengua española en el mundo. Valladolid: Universidad de Valladolid.

Moreno Fernández, F. (2000). ¿Qué español enseñar? Madrid: Arco Libros. 
Variación diatópica del español americano a través de recursos hipermedia

Moreno Fernández, F. (2010). La lengua española en su geografía. Madrid: Arco Libros.

Moreno García, C. (2000). Normas para ser ric@y seguir comunicándose. Recuperado de http:// cvc.cervantes.es/ensenanza/biblioteca ele/asele/ pdf/11/11 0527.pdf

Paricio, M. (2004). Dimensión intercultural en la enseñanza de las lenguas y formación del profesorado. Revista Iberoamericana de Educación, 34(4). Recuperado de http://www.rieoei.org/didactica7.htm

Pastor Cesteros, S. (2016). Enseñanza de español como lengua extranjera. En J. Gutiérrez-Rexach (Ed.), Enciclopedia de Lingüística Hispánica. Nueva lorque: Routledge.

Pfander, S. (2009). Gramática mestiza. Con referencia al castellano de Cochabamba. La Paz: Instituto Boliviano de Lexicografía y otros Estudios Lingüísticos.

Pontes, V. (2009). Abordagem das categorias verbais de tempo, aspecto e modalidade por livros didáticos de língua portuguesa e de língua espanhola: uma análise contrastiva. Fortaleza: Universidade Estadual do Ceará.

Regueiro Rodríguez, M. (2009). Lengua, variación, contextos de enseñanza y qué español enseñar. La variación léxica. Actas del X Encuentro Práctico del profesorado de ELE: Jornadas didácticas del español como lengua extranjera. Madrid: Edinumen International House.

Regueiro Rodríguez, M. (2016). El profesor de ELE y la Didáctica del léxico hispanoamericano. Comprensión frente a infortunio comunicativo. Comunicación presentada en el XXVII Congreso Internacional de ASELE, La Rioja.

Rodrigues, D. (2005). O tratamento da variação linguística em livros didáticos de Língua Inglesa. Fortaleza: Universidade Estadual do Ceará.

Roña, J. (1965). El dialecto "fronterizo" del norte del Uruguay. Montevideo: Librería Anticuaría Americana.

Rosenblat, A. (1984). Estudios sobre el español de América. Caracas: Monte Ávila Editores.
Salvador, G. (2001). El español en España y el español en América. Nueva Revista, marzo-abril, 5860.

Sueiro Justel, J. (2011). Libros de notas, confesionarios, doctrinas y catecismos, poemas y obras de teatro, entre otros textos para la enseñanza/ aprendizaje de lenguas en la lingüística misionero-colonial: el caso de Filipinas. En J. Santiago Guervós, H. Bongaerts, J. Sánchez Iglesias y M. Seseña Gómez (Coords.), Del texto a la lengua: La aplicación de los textos a la enseñanza-aprendizaje del español L2-LE. Salamanca: ASELE.

Torres Solórzano, C. (18 de septiembre de 2010). Choque de lenguas o el mestizaje de nuestro idioma. La Prensa.

Tesoro, P. (2013). El español de los Andes: ¿Variedad estable o español bilingüe? Perspectivas latinoamericanas, 10, 116-132.

Vaquero, M. (1995). El español de América I. Pronunciación. Madrid: Arco Libros.

Vaquero, M. (1996). El español de América II. Morfosintaxis y léxico. Madrid: Arco Libros.

Valls Campà, L. (2004). Enseñanza/aprendizaje de la competencia comunicativa intercultural y análisis de actitudes. Revista marcoELE, 13. Recuperado de http://marcoele.com/descargas/13/valllscompetencia.intercultural.pdf 